\title{
SYNTHESIS AND PHYSICO-CHEMICAL STUDIES OF HETEROMETALLIC NITRILOTRIACETATES OF CHROMIUM(III) WITH SOME 3d METALS
}

\author{
Victor Ciornea*, Aurelian Gulea \\ LCC, Department of Inorganic and Physical Chemistry, Moldova State University, \\ 60 Mateevici str., Chisinau, MD 2009, Moldova \\ *E-mail: ciornea@usm.md,tel. (+37322) 57 75 40,fax. (+373 22) 244248
}

\begin{abstract}
Heterometallic complexes of chromium (III) with some $3 d$ metals have been synthesized based on nitrilotriacetic acid $\left(\mathrm{H}_{3}\right.$ nta), like $\mathrm{M}(b p y)_{2} \mathrm{Cr}_{2}(\mathrm{OH})_{2}(n t a)_{2} \cdot \mathrm{nH}_{2} \mathrm{O}$, where $\mathrm{M}=\mathrm{Mn}^{2+}, \mathrm{Co}^{2+}, \mathrm{Ni}^{2+}$ and $\mathrm{Zn}^{2+} ;$ bpy $=\alpha, \alpha^{\prime}-$ bipyridine; $\mathrm{n}=8$ or 9 . Their chemical composition has been determined from the results of the elemental analysis and thermogravimetric study. The coordination modes of the $n t a^{3-}$ ligand and the type of chemical bonds have been proposed basing on IR spectra.
\end{abstract}

Keywords: heterometallic, nitrilotriacetates, chromium(III), $3 d$ metals, bipyridine.

\begin{abstract}
Introduction
The scientific and technological interest for new materials by means of complex combinations is explained suggestively having as objective the obtaining of new materials with useful properties. Complex compounds are perspective precursors for the synthesis of materials with catalytic [1-3], electric [4-6] and magnetic [7-9] properties. In this sense chromium (III) compounds came to be promising in the design of magnetic materials [10-17]. Thus, depending on basic ligands, the bridging and contraions ones from outer sphere, compounds with ferro- and antiferromagnetic [7-23] properties are obtained.

Taking into consideration the high interest for this type of compounds systemic investigations have been proposed to be carried out that will allow the enlarging of the knowledge about heterometallic combinations of chromium (III) with some elements from $3 \mathrm{~d}$ blocs based on nitrilotriacetic acid $\left(\mathrm{H}_{3} n t a\right)$.
\end{abstract}

\section{Results and Discussions}

Heterometallic complexes of chromium (III) with some $3 d$ metals have been synthesized based on nitrilotriacetic acid $\left(\mathrm{H}_{3} n t a\right)$, like $\mathrm{M}(\text { bpy })_{2} \mathrm{Cr}_{2}(\mathrm{OH})_{2}(n t a)_{2} \cdot \mathrm{nH}_{2} \mathrm{O}$, where $\mathrm{M}=\mathrm{Mn}^{2+}[1], \mathrm{Co}^{2+}[2], \mathrm{Ni}^{2+}[3]$, and $\mathrm{Zn}^{2+}[4]$, bpy $=\alpha, \alpha^{\prime}$-bipyridine; $\mathrm{n}=8$ or 9 were prepared by exchange reaction between the complex salt $\mathrm{Ba}\left[\mathrm{Cr}_{2}(\mu-\mathrm{OH})_{2}(\text { nta })_{2}\right] \cdot 5 \mathrm{H}_{2} \mathrm{O}[24]$ and the respective sulfates on the basis of $\alpha, \alpha^{\prime}$-bipyridine. The reactions proceed in aqueous solutions.

The composition of compounds 1-4 was determined from the elemental, thermogravimetric analysis (table 1), IR(table 2) and UV-Vis spectroscopy.

The electronic absorption spectra recorded for the aqueous solutions of compounds 1-4 show 3 bands. Together with the two bands, $\left.v_{1}{ }^{4} \mathrm{~A}_{2 \mathrm{~g}} \rightarrow{ }^{4} \mathrm{~T}_{2 \mathrm{~g}}\right)=17422 \mathrm{~cm}^{-1}\left(\varepsilon=147.51 \cdot \mathrm{mol}^{-1} \cdot \mathrm{cm}^{-1}\right)$ and $\left.v_{2}{ }^{4} \mathrm{~A}_{2 \mathrm{~g}} \rightarrow{ }^{4} \mathrm{~T}_{1 \mathrm{~g}}\right)=24876 \mathrm{~cm}^{-1}\left(\varepsilon=1651 \cdot \mathrm{mol}^{-1} \cdot \mathrm{cm}^{-1}\right)$ characteristic for $\left\{\mathrm{NO}_{5}\right\}$ octahedral coordination for $\mathrm{Cr}(\mathrm{III})$ with $n t a^{3-}$ ligand, which are also present in the spectra of the complexes $\mathrm{MCr}_{2}(\mathrm{OH})_{2}(n t a)_{2} \cdot \mathrm{nH}_{2} \mathrm{O}$ described in [25], appears one more signal at $v_{3}\left(\pi \rightarrow \pi^{*}\right)=31847 \mathrm{~cm}^{-1}(\varepsilon=900$ $\left.1 \cdot \mathrm{mol}^{-1} \cdot \mathrm{cm}^{-1}\right)$.These parameters confirm the idea that the complexes 1-4 dissociate in solutions but keep the anionic structure unchanged $[25,26]$.

The analysis of the IR spectra showed that the spectra of the compounds 1-4 are identical having unimportant vibrations of the signals shift. The spectrum of the compound $\mathbf{1}$ is a little different (table 2).

In the spectrum of the compound 1 a very intense signal appears at $1606 \mathrm{~cm}^{-1}$ with a shoulder at $1627 \mathrm{~cm}^{-1}$ and three signals appear at 1386, 1344 and $1312 \mathrm{~cm}^{-1}$ related to $v_{\mathrm{s}}\left(\mathrm{COO}^{-}\right)$. The differences $\Delta v=v_{\text {as }}\left(\mathrm{COO}^{-}\right)-v_{\mathrm{s}}\left(\mathrm{COO}^{-}\right)$are equal to 220, 262 and $294 \mathrm{~cm}^{-1}$ (for the first signal) and 241, 283 and $315 \mathrm{~cm}^{-1}$ (shoulder signal). According to [27-29] $\Delta v$ differences higher than $250 \mathrm{~cm}^{-1}$ indicate that $\mathrm{COO}^{-}$groups are monodentate, but differences lower than $250 \mathrm{~cm}^{-1}$ indicate that these groups are bidentate; but if $\Delta v>225 \mathrm{~cm}^{-1}$ the bonds are predominantly covalent and if $\Delta v<225 \mathrm{~cm}^{-1}$ the bonds are predominantly ionic. Taking into consideration these data two coordination modes of the $\mathrm{COO}^{-}$groups are realized in compound $\mathbf{1}$ : two are monodentate having predominantly covalent $\mathrm{M}-\mathrm{O}\left(\mathrm{COO}^{-}\right)$bonds and a $\mathrm{COO}^{-}$ group is bidentate having intermediary covalent-weak ionic $\mathrm{M}-\mathrm{O}\left(\mathrm{COO}^{-}\right)$bond [29]. In the spectra of compounds 2-4 $v_{\text {as }}\left(\mathrm{COO}^{-}\right)$signals appear at $c a 1605(2), 1606(3)$ and $1605 \mathrm{~cm}^{-1}(4)$, but $v_{\mathrm{a}}\left(\mathrm{COO}^{-}\right)$signals appear at 1384 (with shoulder), $1318 \mathrm{~cm}^{-1}$ (2), 1384, 1342, $1318 \mathrm{~cm}^{-1}$ (3) and 1384 (with shoulder), $1319 \mathrm{~cm}^{-1}$ (4). Differences $\Delta v=221,287$ $\mathrm{cm}^{-1}$ (2), 221, 264, $288 \mathrm{~cm}^{-1}$ (3) and 221, $286 \mathrm{~cm}^{-1}$ (4). These $\Delta v$ values are the result of the similar $\mathrm{COO}^{-}$groups coordination of these compounds (2-4) by forming predominantly covalent bonds of the $\mathrm{M}-\mathrm{O}\left(\mathrm{COO}^{-}\right)$bonds and of some weak-ionic bonds of the $\mathrm{M}-\mathrm{O}\left(\mathrm{COO}^{-}\right)$bonds. It could be supposed that the appearance of such low values $\Delta v \sim 221$ $\mathrm{cm}^{-1}$ find the explanation in the existence of $\mathrm{H} . . \mathrm{O}\left(\mathrm{COO}^{-}\right)$bonds, values that cause the diminution of the order of $\mathrm{M}-$ $\mathrm{O}\left(\mathrm{COO}^{-}\right)$bonds from covalent to weak-ionic. Hydrogen bonds (intermolecular, three-dimensional) appear in the IR spectra as strong absorption bands with maximum at $3217 \mathrm{~cm}^{-1}(\mathbf{1})$ and $3318 \mathrm{~cm}^{-1}(\mathbf{2 - 4})$. 
The IR spectra of compounds $\mathbf{2 - 4}$ showed three bands $\delta_{(\mathrm{O}-\mathrm{C}-\mathrm{O})}, \rho_{\omega(\mathrm{O}-\mathrm{C}-\mathrm{O})}$ and $\pi_{(\mathrm{O}-\mathrm{C}-\mathrm{O})}$. This phenomenon is due to the fact that the donor carboxylic groups contribute to the formation of $\mathrm{M}-\mathrm{O}\left(\mathrm{COO}^{-}\right)$groups. The fact that a $\mathrm{COO}^{-}$group of the $n t a^{3-}$ ligand is fixed between two coordination centers leads to the lack of a $\rho_{\omega(\mathrm{O}-\mathrm{C}-\mathrm{O})}$ signal in the spectra of compound $\mathbf{1}$ unlike the series 2-4.

Absorption band $\delta_{(\mathrm{Cr}-\mathrm{O}-\mathrm{H})}$ appeared in the IR spectra at 913 (1), 910 (2), 909 (3) and 910 (4) $\mathrm{cm}^{-1}$ as a very intense signal. The thermogravimetric analyses of compounds 2-4 showed that these compounds are subdued to some thermal transformation while heating (table 1). Dehydration and deaquation processes proceeded over the range $20-180^{\circ} \mathrm{C}$ in two steps with maximum for the first step $c a 70-100^{\circ} \mathrm{C}$ and for the second step at $c a 120-180^{\circ} \mathrm{C}$. Dehydration of $\mathbf{1}$ occurred in one step with the maximum at $100^{\circ} \mathrm{C}$. This different behavior can be explained in the following way: the heating determines the system energy to grow, on the other hand the non-coordinated bipyridine molecules become in such conditions disposed to coordination and substitute the water molecules from the coordination sphere of the Mn atom.

At temperatures over $180-500^{\circ} \mathrm{C}$ thermal decomposition of the organic parts took place. These processes occurred in one step resulting finely in inorganic residues.

\section{Experimental Section}

\section{Synthesis}

[( $\alpha, \alpha^{\prime}$-bipyridyl)-triaqua-manganese(II)-\{di- $\mu$-hydroxy-bis-(nitrilotriacetato)dichromium(III) $\left.\}\right]-\left(\alpha, \alpha^{\prime}\right.$-bipyridylate)pentahydrate, $\left[\mathrm{Mn}\left(\mathrm{H}_{2} \mathrm{O}\right)_{3}(\right.$ bpy $\left.) \mathrm{Cr}_{2}(\mathrm{OH})_{2}(\text { nta })_{2}\right] \cdot($ bpy $) \cdot 5 \mathrm{H}_{2} \mathrm{O}(\mathbf{1})$

$0,246 \mathrm{~g}(1 \mathrm{mmol}) \mathrm{MnSO}_{4} \cdot 7 \mathrm{H}_{2} \mathrm{O}$ and $0,32 \mathrm{~g}(2 \mathrm{mmol}) \alpha, \alpha$-bipyridine were dissolved in $30 \mathrm{ml}$ of hot water. A pale yellow solution was obtained. $0,742 \mathrm{~g}(1 \mathrm{mmol})$ crystalline $\mathrm{BaCr}_{2}(n t a)_{2}(\mathrm{OH})_{2} \cdot 5 \mathrm{H}_{2} \mathrm{O}$, prepared according the source [24], was then added. The mixture was then heated and stirred for an hour at a temperature of $40-50^{\circ} \mathrm{C}$. All this gave an intense green-bluish solution, which was filtered and concentrated by evaporation at a temperature of $40-50^{\circ} \mathrm{C}$ to a $20 \mathrm{ml}$ volume. Within 24 hours a green-bluish crystalline substance precipitated from the solution, which was then filtered, washed with cold water, alcohol and then with acetone and dried at room temperature. Yield 75\%. Anal. Found \%: Cr 10.20; C 37.39; H 4.65; N 8.05. Calc. for $\mathrm{MnCr}_{2} \mathrm{C}_{32} \mathrm{H}_{46} \mathrm{~N}_{6} \mathrm{O}_{22}(\mathbf{1 0 2 5 . 6 7}) \%$ : Cr 10.14; C 37.47; H 4.52; N 8.19.

$$
\text { [Di-( } \alpha, \alpha^{\prime} \text {-bipyridyl)-diaqua-cobalt(II)][di- } \mu \text {-hydroxy-bis-(nitrilotriacetato)dichromate(III)] heptahydrate, }
$$

$$
\left[\mathrm{Co}(\text { bpy })_{2}\left(\mathrm{H}_{2} \mathrm{O}\right)_{2}\right]\left[\mathrm{Cr}_{2}(\mathrm{OH})_{2}(\text { nta })_{2}\right] \cdot 7 \mathrm{H}_{2} \mathrm{O}(2)
$$

Compound 2 was prepared according to synthesis $\mathbf{1}$, but the procedure took place in $\mathrm{CO}_{2}$ and $\mathrm{N}_{2}$ environment. After $48 \mathrm{~h}$ a crystalline blue-violet substance crystallized from the solution, which was then filtered, washed with cold water,

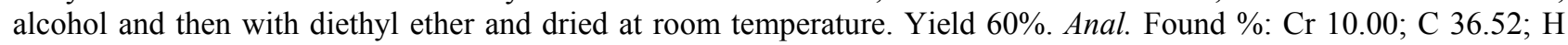
4.50; N 7,90. Calc. for $\mathrm{CoCr}_{2} \mathrm{C}_{32} \mathrm{H}_{48} \mathrm{~N}_{6} \mathrm{O}_{23}$ (1047.68) \%: Cr 9.93; C 36.69; H 4.62; N 8.02.

[Di-( $\alpha, \alpha^{\prime}$-bipyridyl)-diaqua-nickel(II)][di- $\mu$-hydroxy-bis-(nitrilotriacetato)dichromate(III)] heptahydrate, $\left[\mathrm{Ni}(\text { bpy })_{2}\left(\mathrm{H}_{2} \mathrm{O}\right)_{2}\right]\left[\mathrm{Cr}_{2}(\mathrm{OH})_{2}(\text { nta })_{2}\right] \cdot 7 \mathrm{H}_{2} \mathrm{O}(3)$

Compound 3 was prepared according to synthesis 1. A crystalline blue-violet substance was obtained. Yield 68\%. Anal. Found \%: Cr 9.98; C 36.57; H 4.52; N 8.00. Calc. for $\mathrm{NiCr}_{2} \mathrm{C}_{32} \mathrm{H}_{48} \mathrm{~N}_{6} \mathrm{O}_{23}(\mathbf{1 0 4 7 . 4 4 )} \%$ : Cr 9.93; C 36.69; H 4.62; N 8.02 .

[Di-( $\alpha, \alpha^{\prime}$-bipyridyl)-diaqua-zinc(II)][di- $\mu$-hydroxy-bis-(nitrilotriacetato)dichromate(III)] heptahydrate,

$\left[\mathrm{Zn}(\text { bpy })_{2}\left(\mathrm{H}_{2} \mathrm{O}\right)_{2}\right]\left[\mathrm{Cr}_{2}(\mathrm{OH})_{2}(\text { nta })_{2}\right] \cdot 7 \mathrm{H}_{2} \mathrm{O}(4)$

Compound 4 was prepared according to synthesis 1. A crystalline blue-violet substance was obtained. Yield 62\%. Anal. Found \%: Cr 9.70; C 36.65; H 4.47; N 7.95. Calc. for $\mathrm{ZnCr}_{2} \mathrm{C}_{32} \mathrm{H}_{48} \mathrm{~N}_{6} \mathrm{O}_{23}$ (1054.14) \%: Cr 9.87; C 36.46; H 4.59; N 7.97.

Compounds 1-4 are soluble in water, especially at heating; DMSO, DMF soluble; insoluble in methanol, ethanol, acetone and diethyl ether.

\begin{tabular}{|c|c|c|c|c|c|c|c|c|c|}
\hline \multirow{2}{*}{$\mathrm{Nr}$} & \multirow{2}{*}{ Complex } & \multicolumn{6}{|c|}{ Dehydration and deaquation processes, ${ }^{\circ} \mathrm{C}$} & \multicolumn{2}{|c|}{ Thermal decomposition, ${ }^{\circ} \mathrm{C}$} \\
\hline & & $-\mathrm{nH}_{2} \mathrm{O}$ & $\Delta \exp$. & $\Delta$ calc. & Start & Max. & Fin. & Start & Fin. \\
\hline 1 & $\mathrm{Mn}(b p y)_{2} \mathrm{Cr}_{2}(n t a)_{2}(\mathrm{OH})_{2} \cdot 8 \mathrm{H}_{2} \mathrm{O}$ & 8 & 14.5 & 14.05 & 50 & 100 & 120 & 220 & 470 \\
\hline \multirow{2}{*}{2} & \multirow{2}{*}{$\mathrm{Co}(b p y)_{2} \mathrm{Cr}_{2}(n t a)_{2}(\mathrm{OH})_{2} \cdot 9 \mathrm{H}_{2} \mathrm{O}$} & 4 & 6.5 & 6.88 & 50 & 100 & 120 & \multirow{2}{*}{240} & \multirow{2}{*}{460} \\
\hline & & 5 & 9.0 & 8.60 & 120 & 150 & 180 & & \\
\hline \multirow{2}{*}{3} & \multirow{2}{*}{$\mathrm{Ni}(b p y)_{2} \mathrm{Cr}_{2}(n t a)_{2}(\mathrm{OH})_{2} \cdot 9 \mathrm{H}_{2} \mathrm{O}$} & 4 & 6.5 & 6.88 & 40 & 80 & 100 & \multirow{2}{*}{180} & \multirow{2}{*}{470} \\
\hline & & 5 & 9.0 & 8.60 & 100 & 140 & 180 & & \\
\hline \multirow{2}{*}{4} & \multirow{2}{*}{$\mathrm{Zn}(b p y)_{2} \mathrm{Cr}_{2}(n t a)_{2}(\mathrm{OH})_{2} \cdot 9 \mathrm{H}_{2} \mathrm{O}$} & 5 & 8.5 & 8.54 & 20 & 70 & 100 & \multirow{2}{*}{180} & \multirow{2}{*}{430} \\
\hline & & 4 & 7.0 & 6.84 & 100 & 120 & 180 & & \\
\hline
\end{tabular}

Table 1. Thermogravimetric analysis

\section{Chemical and Physicochemical analyses}

The chemical composition was determined from the general methods of $\mathrm{Cr}, \mathrm{C}, \mathrm{H}$ and $\mathrm{N}$ chemical analysis. The water was determined from thermal analysis (table 1).

The thermogravimetric analysis was performed on a Paulik-Paulik-Erday M-102 derivatograph, under air atmosphere, over the temperature range $t_{\text {room }}-800^{\circ} \mathrm{C}$, at $2,5^{\circ} \mathrm{C} / \mathrm{min}$ or $5^{\circ} \mathrm{C} / \mathrm{min}$ speed of tests heating. Compounds 1-4 were 
analyzed by IR spectroscopy, using tests of tablets form in KBr. IR spectra were recorded on a Perkin Elmer FTIR 1650 spectrometer in the range 4000-400 $\mathrm{cm}^{-1}$ at the Institute of Biology and Molecular Chemistry of Lausanne, Switzerland. Data of IR spectra are given in table 2 .

Table 2. IR spectra of the compounds 1-4

\begin{tabular}{|c|c|c|c|c|c|c|}
\hline \multirow{2}{*}{\multicolumn{3}{|c|}{$\begin{array}{c}\text { Complex } \\
\lambda, \mathbf{c m}^{-1}\end{array}$}} & \multirow{2}{*}{1} & \multirow{2}{*}{2} & \multirow{2}{*}{3} & \multirow{2}{*}{4} \\
\hline & & & & & & \\
\hline $\mathrm{H}_{2} \mathrm{O}$ & $v$ & H...OH & 3217 broad, intense & 3318 broad, intense & 3318 broad, intense & $\begin{array}{l}3318 \text { broad, } \\
\text { intense }\end{array}$ \\
\hline $\mathrm{OH}$ & $\delta$ & $\mathrm{Cr}-\mathrm{O}-\mathrm{H}$ & 913 intense & 910 intense & 909 intense & 910 intense \\
\hline \multirow{6}{*}{$\mathrm{COO}^{-}$} & $v_{\text {as }}$ & $\mathrm{C}-\mathrm{O}$ & $\begin{array}{l}1627 \text { (shoulder), } \\
1606 \text { very intense, } \\
\text { shoulder }\end{array}$ & 1605 very intense & $\begin{array}{l}1606 \text { very intense, } \\
\text { shoulder }\end{array}$ & $\begin{array}{l}1605 \text { very } \\
\text { intense }\end{array}$ \\
\hline & $v_{\mathrm{s}}$ & $\mathrm{C}-\mathrm{O}$ & $\begin{array}{l}1386 \text { intense, } 1344, \\
\text { medium, } 1312 \\
\text { medium, split }\end{array}$ & $\begin{array}{l}1384 \text { with two } \\
\text { peaks, intense, } \\
\text { shoulder, } 1318 \\
\text { medium }\end{array}$ & $\begin{array}{l}1384 \text { with two peaks, } \\
\text { intense, shoulder, } \\
1318 \text { medium }\end{array}$ & $\begin{array}{l}1384 \text { intense, } \\
\text { shoulder; } 1319 \\
\text { medium }\end{array}$ \\
\hline & \multicolumn{2}{|c|}{$\Delta \mathrm{v}=\mathrm{v}_{\mathrm{as}}-\mathrm{v}_{\mathrm{s}}$} & $\begin{array}{l}220,241,262,283, \\
294,315\end{array}$ & 221,287 & $222,264,288$ & 221,286 \\
\hline & $\delta$ & $\mathrm{O}-\mathrm{C}-\mathrm{O}$ & $\begin{array}{l}776,745,736 \\
\text { intensely split }\end{array}$ & 748,736 intense & $\begin{array}{l}747,735 \text { split, two } \\
\text { shoulders, intense }\end{array}$ & $\begin{array}{l}736, \text { two } \\
\text { shoulders, } \\
\text { intense }\end{array}$ \\
\hline & $\rho_{\omega}$ & $\mathrm{O}-\mathrm{C}-\mathrm{O}$ & $\begin{array}{l}619 \text { shoulder, } \\
\text { intense }\end{array}$ & $\begin{array}{l}613 \text { shoulder, } \\
\text { intense }\end{array}$ & 613 intense & 613 intense \\
\hline & $\pi$ & $\mathrm{O}-\mathrm{C}-\mathrm{O}$ & $\begin{array}{l}\text { 528, } 514 \text { split, } \\
\text { intense }\end{array}$ & $\begin{array}{l}551 \text { intense, } 522, \\
512 \text { split, intense }\end{array}$ & $\begin{array}{l}551 \text { intense, } 510,503 \\
\text { shoulder, very } \\
\text { intense }\end{array}$ & $\begin{array}{l}550 \text { intense, } 522, \\
513 \text { very intense }\end{array}$ \\
\hline $\mathrm{CN}$ & $v$ & $\mathrm{C}-\mathrm{N}$ & $\begin{array}{l}\text { 1107, } 1096 \text { split, } \\
\text { weak, } 1007 \\
\text { shoulder, medium }\end{array}$ & $\begin{array}{l}1094 \text { with two } \\
\text { peaks, medium, } \\
1022 \text { medium }\end{array}$ & $\begin{array}{l}1105 \text { with two peaks, } \\
\text { medium, } 1025 \\
\text { medium }\end{array}$ & $\begin{array}{l}1105 \text { weakly } \\
\text { split, } 1022 \\
\text { medium }\end{array}$ \\
\hline \multirow{4}{*}{$\mathrm{CH}_{2}$} & $v$ & $\mathrm{C}-\mathrm{H}$ & - weak & 2978 weak & - weak & - weak \\
\hline & $\delta$ & $\mathrm{H}-\mathrm{C}-\mathrm{H}$ & $\begin{array}{l}\text { 1461, } 1426 \text { medium } \\
\text { split }\end{array}$ & - & - & - \\
\hline & $\rho_{\omega}$ & $\mathrm{CH}_{2}$ & 1271,1226 medium & $\begin{array}{l}\text { 1265, split, medium, } \\
1212 \text { medium }\end{array}$ & $\begin{array}{l}\text { 1264, split, medium, } \\
1212 \text { medium }\end{array}$ & $\begin{array}{l}\text { 1265, split, } \\
\text { medium, 1212, } \\
\text { medium }\end{array}$ \\
\hline & $\rho_{\mathrm{r}}$ & $\begin{array}{l}\mathrm{CH}_{2} \\
\text { (gosh) }\end{array}$ & 971 medium & - & - & - \\
\hline $\begin{array}{l}\mathrm{CC} \\
\left(n t a^{3-}\right)\end{array}$ & $v$ & $\mathrm{C}-\mathrm{C}$ & 943 medium & $\begin{array}{l}947 \text { split, shoulder, } \\
\text { medium }\end{array}$ & $\begin{array}{l}947 \text { split, shoulder, } \\
\text { medium }\end{array}$ & $\begin{array}{l}947 \text { two } \\
\text { shoulders, } \\
\text { medium }\end{array}$ \\
\hline $\begin{array}{l}\mathrm{CC} \\
(b p y)\end{array}$ & $v$ & $\mathrm{C}=\mathrm{C}$ & $\begin{array}{l}1492 \text { weak, } 1474 \\
\text { medium, } 1441 \text { split, } \\
\text { medium }\end{array}$ & $\begin{array}{l}1493 \text { medium, } 1443 \\
\text { medium, shoulder }\end{array}$ & $\begin{array}{l}1494 \text { medium, } 1474 \\
\text { medium, } 1444 \\
\text { medium }\end{array}$ & $\begin{array}{l}1493 \text { medium, } \\
1475 \text { medium, } \\
1443 \text { medium, } \\
\text { shoulder }\end{array}$ \\
\hline
\end{tabular}

\section{Conclusions}

1. Four complexes $\mathrm{M}(\text { bpy })_{2} \mathrm{Cr}_{2}(\mathrm{OH})_{2}(n t a)_{2} \cdot \mathrm{nH}_{2} \mathrm{O}$, where $\mathrm{M}=\mathrm{Mn}^{2+}, \mathrm{Co}^{2+}, \mathrm{Ni}^{2+}$ and $\mathrm{Zn}^{2+} ;$ bpy $=\alpha, \alpha^{\prime}$-bipyridine; $\mathrm{n}=8$, or 9 have been synthesized. Their chemical composition has been determined from the results of the elemental analysis and thermogravimetric study.

2. According to the data of UV-Vis spectra it has been supposed that complexes 1-4 dissociate in solution but keep the anionic $\left[\mathrm{Cr}_{2}(\mathrm{OH})_{2}(n t a)_{2}\right]^{2-}$ structure unchanged.

3. According to the data of the IR spectra two coordination modes of the $\mathrm{COO}^{-}$groups are realized in complex 1: two are monodentate having predominantly covalent $\mathrm{M}-\mathrm{O}\left(\mathrm{COO}^{-}\right)$bonds and one $\mathrm{COO}^{-}$group is bidentate having intermediary covalent-weak ionic $\mathrm{M}-\mathrm{O}\left(\mathrm{COO}^{-}\right)$bond. IR spectra of the complexes 2-4 show that the coordination mode of the $n t a^{3-}$ ligand is identical, $\mathrm{M}-\mathrm{O}\left(\mathrm{COO}^{-}\right)$bonds have predominantly covalent character and one weakionic character of the $\mathrm{M}-\mathrm{O}\left(\mathrm{COO}^{-}\right)$. The latter is the result of the existence of $\mathrm{H} . . \mathrm{O}\left(\mathrm{COO}^{-}\right)$bonds.

4. Thermal analysis of the complexes 1-4 show that at temperatures over $180^{\circ} \mathrm{C}$ they loose the hydration and coordination water molecules, but at temperatures over $180-500^{\circ} \mathrm{C}$ the thermal decomposition of the organic parts takes place. 


\section{Acknowledgments}

This work was supported by Superior Council for Sciences and Technology Developing of the R. Moldova (Project 569-2004) and National Program „Nanotechnologies, new multifunctional materials and electronic microsystems”, registration number 0104MD02481.

\section{References}

[1] Gulea A., et al. / 52 ${ }^{\mathrm{ND}}$ World Exhibition of Innovation Research and New Technology, Brussels, Eureka, 1116/11/2003, Book of Abstracts, sec. 7, p. 76.

[2] Gibson V. et al. // J. Chem. Soc., Dalton Trans., 2002, 4017-4023.

[3] Ruck R. Jacobsen E. // J. Amer. Chem. Soc., 2002, 124, 12, 2882-2883.

[4] V. Tapcov, A. Gulea, V. Stavila. / Moldavian Patent MD2146. BOPI, no. 4, 2003.

[5] Gulea A., et al. / Moldavan Patent MD1559. BOPI no. 11, 2000.

[6] Myung S.-T., et al. // J. Power Sources, 2003, 119-121, 211-215.

[7] Kahn O. “Molecular Magnetism”, New York: VCH, 1993. 380p.

[8] Coronado E., et al. // J. Solid State Chemistry, 2001, 159, 391-402.

[9] Hua Xi., et al. // New J. Chem., 2001, 25, 875-878.

[10] Andruh M., et al. // Inorg. Chim. Acta, 1996, 251, 309-317.

[11] Stanica N., et al. // Polihedron, 1998, 17, 10,1787-1789.

[12] Muñoz C., et al. // J. Chem. Soc., Dalton Trans., 1998, 3125-3131.

[13] Marinescu G., et al. // New J. Chem., 2000, 24, 527-536.

[14] Lescouëzec R., et al. // New J. Chem., 2001, 25, 1224-1235.

[15] Costisor O., et al. // Inorg .Chim. Acta, 2001, 324, 352-358.

[16] Marinescu G., et al. // Inorg. Chim. Acta, 2002, 336, 46-54.

[17] Chiozzone R., et al. // Inorg. Chem., 1999, 38, 4745-4752.

[18] Fujihara Ta., Fuyuhiro Ak., Kaizaki Su. // Inorg. Chim. Acta, 1998, 278, 15-23.

[19] Ross S., et al. // Inorg. Chim. Acta, 2002, 339, 71-76.

[20] Green Ch., et al. // Inorg. Chim. Acta, 1990, 176, 87-93.

[21] Fujihara Ta., Fuyuhiro Ak., Kaizaki Su. // J. Chem. Soc., Dalton Trans., 1995, 1813-1821.

[22] Ni-iya K., et al. // Bull. Chem. Soc. Japan., 2001, 74, 1891-1897.

[23] Kawamura Ya., et al. // Inorg. Chim. Acta, 2004, 357, 2437-2440.

[24] Victor Ciornea // Anal. Ştiinț. USM, ser. "Şt. chim.-biol.”, 2004, 425-431.

[25] Novitchi G., et al. // Eur. J. Inorg. Chem., 2005, 929-937.

[26] V. Ciornea, et al. / XXII Межд. Чугаевская конф. по коорд. химии, 20-24 июня 2005, г. Кишинёв. Тезисы докладов. с. 226.

[27] Накамото К. “ИК-спектры и спектры КР неорганических и комплексных соединений” М., Мир, 1991 ; сс. 259-271.

[28] G. B. Deacon, R. J. Phillips // Coord. Chem. Rev., 1980, 33, 227.

[29] „Координационная химия редкоземельных элементов” М., Изд-во Моск. Ун-та, 1979; сс. 76-101. 